\title{
Feinster Sprühnebel ermöglicht hohe Deposition
}

— In der Vergangenheit ging man davon aus, dass sich das entzündliche Geschehen vorwiegend in den großen und mittleren Atemwegen abspielt. Mittlerweile weiß man aber, dass auch die kleinen, peripheren, aber flächenmäßig bei weitem größten Atemwege von der Entzündung betroffen sind, erklärte Dr. med. Thomas Voshaar, Moers. Bei schwerem Asthma

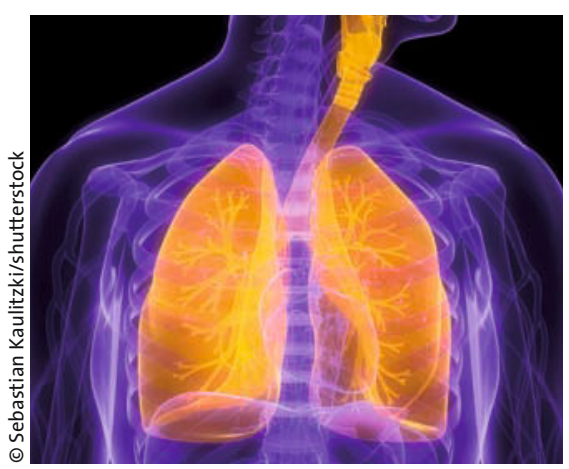

Bei schwerem Asthma ist die Entzündung in den peripheren Atemwegen ausgeprägt. scheint gerade dort die Entzündung besonders ausgeprägt zu sein.

In Studien hat sich gezeigt, dass trotz des Einsatzes inhalativer Kortikosteroide viele Asthma-Patienten eine persistierende Symptomatik mit häufigen Exazerbationen aufweisen.,Offenbar erreichen wir oftmals mit unserer Behandlung die Entzündung in den kleinen Atemwege nicht", betonte Voshaar.

\section{Wirkstoffpartikel erreichen auch} die kleinen Atemwege

Gute Chancen, mit inhalativen Glukokortikoiden als antiinflammatorischer Therapie auch in diese Bereiche vorzudringen, bestehen nur mit besonders kleinen Wirkstoffpartikeln. INUVAIR ${ }^{\circledR}$ Mikrosol, die Fixkombination aus dem inhalativen Glukokortikoid Beclometason und dem Beta-2-Agonisten Formoterol, erfüllt diese Anforderung. Die einzelnen Partikel haben nur eine Größe zwischen ein und zwei Mikrometern und gelangen so bis in entfernte Areale der Lunge.

\section{Einfache Anwendung erleichtert Therapie}

Dr. med. Harald Mitfessel, Remscheid, hat drei Jahre praktische Erfahrungen mit dieser Fixkombination für die Behandlung des mittel- bis schwergradigen, persistierenden Asthma bronchiale. Der Arzt für Lungen- und Bronchialheilkunde unterstrich die einfache Anwendung. Die Wirkstoffe liegen in Lösung vor und müssen nicht geschüttelt werden. Die Fixkombination ist zudem in ihrer klinischen Wirkung der Gabe der Einzelsubstanzen überlegen. Bei jedem Sprühstoß entsteht eine langsame und lang anhaltende feine Sprühwolke, die auch bei niedrigem Atemfluss die Inhalation einer konstanten Dosis gewährleistet.

$\mathrm{mb}$

3. Janssen Pneumocolleg „3 Jahre INUVAIR ${ }^{\oplus}$ Mikrosol: Ein pneumologisches Update", am 27. Oktober 2010 in Düsseldorf (Veranstalter: Janssen-Cilag/Nycomed)

\section{Gezielt gegen die antiinflammatorische Komponente der COPD}

— Die COPD ist ein heterogenes Krankheitsbild mit verschiedenen Phänotypen und einem breiten Spektrum von Komorbiditäten. Da viele Patienten trotz der verfügbaren Therapieoptionen symptomatisch bleiben und häufige Exazerbationen erleben, besteht ein Bedarf nach Medikamenten mit neuartigen Wirkmechanismen, forderte Prof. Peter Barnes, London. Ein besonders Problem sei die COPD-spezifische Entzündung, die auf Kortikosteroide nicht anspricht. Die Entzündungsprozesse bleiben nicht auf die Atemwege beschränkt, sondern können auch andere Organsysteme erfassen und die typischen Komorbiditäten der COPD auslösen.

\section{Neuer antientzündlicher Wirkansatz}

Mit dem PDE-4-Hemmer Roflumilast $\left(\right.$ Daxas $^{\circledR}$ ) steht nun erstmals eine Therapieoption zur Verfügung, die sich gezielt gegen die inflammatorische Komponente der COPD richtet.
Der neue Wirkstoff wurde in vier randomisierten, placebokontrollierten Studien mit insgesamt knapp 5000 COPD-Patienten über sechs oder zwölf Monate untersucht. In die beiden 12-Monats-Studien wurden rund 3000 Patienten mit schweren Atemwegsobstruktionen (postbronchodilatatorisches $\mathrm{FEV}_{1}$ $\geq 50 \%$ ) und mindestens einer Exazerbation im vorausgegangenen Jahr eingeschlossen. Eine Standardtherapie mit langwirksamen Betaagonisten war erlaubt.

\section{Exazerbationsrate gesenkt, Lungenfunktion verbessert}

Die gepoolten Daten dieser beiden Studien zeigen laut Prof. Fernando J. Martinez, Ann Arbor/USA, dass Roflumilast ( $500 \mu \mathrm{g} / \mathrm{d}$ ) zu einer statistisch signifikanten Reduktion der Exazerbationen und zu einer Verbesserung der Lungenfunktion führt.

Die Reduktion der Exazerbationsrate zeigte sich bei allen Patienten, war jedoch beson- ders ausgeprägt bei Patienten, die in den zurückliegenden Monaten zwei und mehr Exazerbationen erlitten hatten. Außerdem verlängerte der PDE-4-Hemmer die Zeit bis zur ersten ( $H R O, 89, p=0,019)$, zweiten ( $H R$ $0,79, p=0,001)$ und dritten Exazerbation (HR 0,73, $P=0,003)$ signifikant im Vergleich zu Placebo.

Eine deutliche Abnahme der Exazerbationen um 20,7\% ( $p=0,001)$ wurde auch beobachtet, wenn Roflumilast zusätzlich zu einem langwirksamen Betaagonisten verabreicht wurde. Als häufigste Nebenwirkungen wurden eine leichte Nausea, Diarrhö und Gewichtsabnahmen berichtet, insgesamt wurde die Substanz aber gut vertragen.

aa

Satellitensymposium „A deeper look into COPD“ im Rahmen des ERS-Kongresses am 19. September 2010 in Barcelona (Veranstalter: Nycomed) 